\title{
Transportation Mode Inference Using Environmental Constraints
}

\author{
Hidekazu Kasahara \\ Kyoto University \\ Yoshida Nihonmatsu-cho, \\ Sakyo-ku \\ Kyoto, Japan 606-8501 \\ hidekazu.kasahara@ \\ mm.media.kyoto-u.ac.jp
}

\author{
M. liyama \\ Kyoto University \\ Yoshida Nihonmatsu-cho, \\ Sakyo-ku \\ Kyoto, Japan 606-8501 \\ iiyama@mm.media. \\ kyoto-u.ac.jp
}

\author{
M. Minoh \\ Kyoto University \\ Yoshida Nihonmatsu-cho, \\ Sakyo-ku \\ Kyoto, Japan 606-8501 \\ minoh@mm.media. \\ kyoto-u.ac.jp
}

\begin{abstract}
Analysis of transportation mode used by tourists in touristic destination areas provides basic information of tourism policy making for local governments or marketing strategy making for the tourism industry. With the technical advances of tracking devices, GPS supported smartphones sense the movement of tourists, and generate a large volumes of data which show tourists' trajectory data. Because GPS trajectory data has only latitudes, longitude and time, transportation modes should be inferred by any methods. Some researchers infer transportation modes from velocity of tourists by using machine learning like Support Vector Machine (SVM) and Conditional Random Field (CRF). However, because trains and buses temporarily stop at stations or bus stops, respectively, when movement is slow, the transportation mode can not be inferred correctly only from velocity. The locations where trains and buses temporarily stop are generally known in advance. Therefore, the transportation mode can be correctly inferred by using such location data as environmental constraints.In this research, we propose a new transportation mode inference method using environmental constraints. We assume that tourists move by foot or public transportation in the large-size touristic destinations which include many touristic spots.
\end{abstract}

\section{CCS Concepts}

-Information systems $\rightarrow$ Global positioning systems; Data mining; •Computing methodologies $\rightarrow$ Machine learning approaches;

\section{Keywords}

GPS; transportation mode; environmental factor; pattern recognition; machine learning

\section{INTRODUCTION}

In this paper, transportation mode is inferred by analyzing tourists' movement trajectories sensed at regular intervals within environmental constraints. We define a human movement trajectory sensed

\footnotetext{
Permission to make digital or hard copies of all or part of this work for personal or classroom use is granted without fee provided that copies are not made or distributed for profit or commercial advantage and that copies bear this notice and the full citation on the first page. Copyrights for components of this work owned by others than ACM must be honored. Abstracting with credit is permitted. To copy otherwise, or republish, to post on servers or to redistribute to lists, requires prior specific permission and/or a fee. Request permissions from permissions@acm.org.

IMCOM '17, January 05-07, 2017, Beppu, Japan

(C) 2017 ACM. ISBN $978-1-4503-4888-1 / 17 / 01 \ldots \$ 15.00$

DOI: http://dx.doi.org/10.1145/3022227.3022309
}

at regular intervals as a consecutive trajectory. A consecutive trajectory is obtained from GPS-measured location data. A semantic description of the transportation mode used for a consecutive trajectory is necessary for understanding a tourist's activity.

By considering a tourist's transportation modes, he/she can be provided with adequate evacuation advice. For example, if a tourist rides on the train, the nearest station is the best evacuation place, not the nearest evacuation place. Evacuation support based on the understanding of the transportation mode efficiently allocates social resources allocation during a disaster situation. In non-disaster situations, route recommendation that considers a tourist's transportation usage helps alleviate the concentration of tourists by avoiding routes with crowded transportation. In addition, traffic statistics are calculated to assist the creation of local government tourism policies. Understanding a tourist activity is important for the tourism industry and the local government of the tourism destination when creating tourism policy or marketing strategy.

Traditional questionnaires, face-to-face interviews, and traffic analysis are frequently employed for tracking tourist activity including transportation mode tracking. With the technical advances of sensors, GPS supported smartphones can sense tourist activity and generate large volume of consecutive trajectories. However, these consecutive trajectories lack semantic richness because they include only coordinates and time. To enhance tourist activity analysis, the semantic richness of the data should be improved.

Many researchers have studied the transportation mode inference using machine learning $[2,4]$. However, because trains and buses temporarily stop at stations or bus stops, respectively, when movement is slow, the transportation mode can not be inferred correctly from velocity. However, the locations where the train and bus temporarily stop are generally known. Therefore, the transportation mode can be correctly inferred by using such location data as environmental constraints. This paper proposes a novel method to infer the transportation mode from consecutive trajectories using environmental constraints.

This paper is organized as follows. In Section 2, related research is reviewed. In Section 3, the proposed method is described. The experiments are described in Section 4, and the results are presented in Section 5. In Section 6, these results are discussed. This paper is concluded and future directions for this work are discussed in Section 7.

\section{RELATED RESEARCH}

Many studies have investigated transportation mode inference. The approaches of the existing research can be categorized into two types: those based on the features of an observed object, and 
those that use features surrounding the observed object. The first approach is defined as an object feature approach, and the second one is defined as an environmental feature approach.

The object feature approach estimates the transportation modes by learning the features in the training data. Some studies have proposed the employment of decision trees $[1,16]$, hierarchical conditional random fields [7], and support vector machines (SVM) [2, 15, 17]. Kinoshita et al. discussed the application of a latent statistical model to moving mode estimation and interpolation of the GPS observation [6]. However, as stated in Section 1, this approach is unable to distinguish some modes using velocity only in the case of occasional deceleration. In contrast to researchers that use velocity, Ohashi proposed a transportation mode inference method that uses the fluctuation of movement, measured by an accelerometer that is equipped in smartphones [10]. In addition, Hemminki inferred the transportation mode using an adaptive boost method and acceleration data measured by a three-axis accelerator [4]. These types of sensor data are difficult to collect. GPS trajectory data is used in this research because it is available in a broad range of wearable devices, and it is easy to collect.

Regarding the environmental feature approach, some researchers have proposed methods that use spatial data such as transportation networks or land use data $[8,11,12,14]$.

Yan et al divide the GPS trajectory data into moving episodes and stopping episodes, and infer the activities of each episode [14]. A moving episode is inferred to be a transportation mode, and a stopping episode is inferred to be an event. In this method, the input is raw GPS trajectory data, and after the system corrects for the error, it divides the GPS trajectory using temporal-spatial features such as velocity, acceleration, and movement direction. The system infers the transportation mode of a moving episode and the activity of the stopping episode using outside knowledge, such as road type or land usage, and outputs the results. Yan focuses on inferring the activity of the stopping episode, and he does not describe the details of the transportation mode inference. It is possible that he simply infers the transportation mode from the corresponding road type.

Stenneth et al proposed an inference method using GIS information that belongs to the environment of the moving objects such as real-time public bus locations, railway networks, and bus stops [12]. For the real-time public bus locations, he used the real-time location data of all the public buses in Chicago, USA. He infers the transportation mode of each observation point using features for random forest machine learning that include average bus closeness $(\mathrm{ABC})$, candidate bus closeness $(\mathrm{CBC})$, rail line trajectory closeness, bus stop closeness rate, average velocity, average heading change, average acceleration, and GPS accuracy. He calculates the $\mathrm{ABC}$ feature as the average Euclidean distance between the object and the nearest bus, and the $\mathrm{CBC}$ feature as the smallest total of Euclidean distance between the object and each bus. They improve the classification effectiveness using machine learning by identifying and deriving the relevant features related to transportation network information. However, the reason why ABC or GPS accuracy are adopted is not explained. In addition, if the real time public bus location in a certain area is known, the bus that the object rides on can be identified, and transportation mode inference is of no practical use. However, his research does not take into consideration such characteristics of real time data.

The proposed method in this paper infers the transportation mode from a GPS trajectory using different inference standards corresponding to the position of the object.

\section{MODE INFERENCE BY ENVIRONMEN- TAL CONSTRAINTS}

\subsection{Problem Setting}

A GPS trajectory is defined as time series data consisting of observation points $i\left(x_{i}, y_{i}, t_{i}\right)$ (where $x, y$ are the coordinates and $t$ is time). In this research, the tourist's GPS trajectory data is input into the system and the system outputs a set of sequential data consisting of multiple transportation modes $m \in M$ (eq.1) of the tourist's movement:

$$
M=\left\{m_{1}, m_{2}, m_{3}, \cdots, m_{n}\right\}
$$

Here, $M$ generally includes walking, bicycle, motorbike, public bus, taxi, rental car, private car, and train modes. Some modes are not available in some areas. The transportation modes, which are the target of the inference, depend on the users of the inference results in the survey area. In this research, the transportation modes that are out of scope are lumped into one transportation mode because all observation points are labeled.

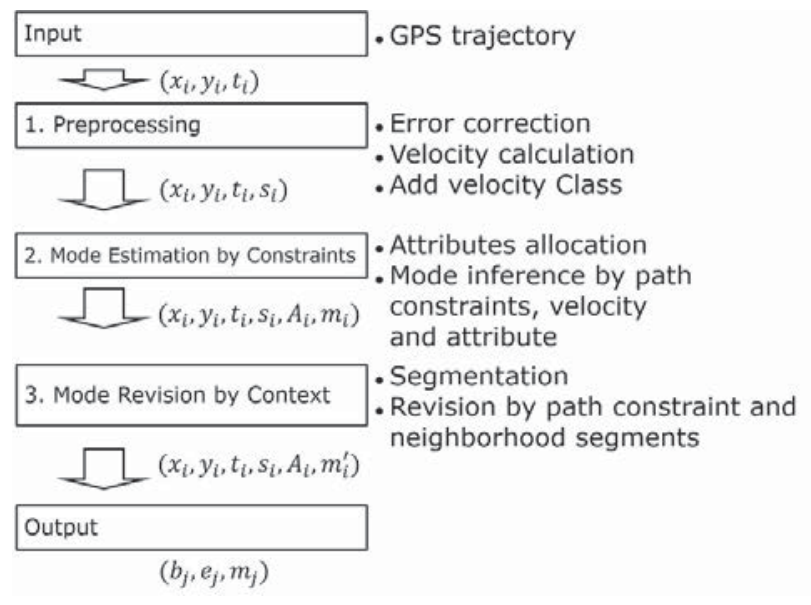

Figure 1: Overview of the Proposed Method

\subsection{Environmental Constraints}

Movement in physical space is restricted by some factors that exist in the environment. The range of velocity is constrained depending on the time and location. The possible path for some transportation modes is limited, for example, for train and bus routes. In this research, the restrictions on the movement that exist in the environment are defined as environmental constraints $e$.

An environmental constraint $e$ can be given as a point, line, or area in physical space. Examples of point constraints are a railway station or bus stop. Examples of linear constraints are a road, railway, footpath, or cycling path, and examples of area constraints are a tourism spot or car park. These environmental constraints are not disjoint, and some environmental constraints can be allocated to the same point, like a railway and one of its stations. Therefore, we allocate the set $A_{i}=\left\{a_{1}, a_{2}, a_{3}, \ldots, a_{l}\right\}$, which indicates the existence of all environmental constraints at each observation point of the GPS trajectory data, where $l$ is the number of environmental constraints.

When inferring transportation mode $m$ from the observation points of GPS trajectory data, the change of velocity that is caused by the environmental constraints $e$ should be considered. Without this consideration, $m$ cannot be correctly inferred. The velocity of available transportation modes given various environmental constraints 
Table 1: Velocity of Available Transportation Modes Given Environmental Constraints

\begin{tabular}{|c|c|c|c|c|c|c|c|c|c|c|}
\hline & & \multicolumn{9}{|c|}{ Environmental Constraints $e$} \\
\hline & & \multicolumn{2}{|c|}{ Point } & \multicolumn{5}{|c|}{ Line } & \multicolumn{2}{|c|}{ Area } \\
\hline & & \multirow[b]{2}{*}{ Stop } & \multirow{2}{*}{ Crossing } & \multicolumn{2}{|c|}{ Car Road } & \multirow[b]{2}{*}{ Rail } & \multirow[b]{2}{*}{$\begin{array}{l}\text { Walk } \\
\text { Road }\end{array}$} & \multirow[b]{2}{*}{$\begin{array}{l}\text { Bicycle } \\
\text { Road }\end{array}$} & \multirow[b]{2}{*}{ Spot } & \multirow[b]{2}{*}{ Car park } \\
\hline & & & & Bus Route & Others & & & & & \\
\hline \multirow{6}{*}{$\begin{array}{l}\text { Mode } \\
m\end{array}$} & Walking & L & L & $\mathrm{L}$ & L & - & $\mathrm{L}$ & - & $\mathrm{L}$ & L \\
\hline & Bicycle & L-M & L-M & L-M & L-M & - & - & L-M & - & $\mathrm{L}$ \\
\hline & Motorbike & $\mathrm{H}$ & L-H & $\mathrm{H}$ & $\mathrm{H}$ & - & - & - & - & $\mathrm{L}$ \\
\hline & Bus & $\mathrm{L}$ & L-H & $\mathrm{H}$ & $\mathrm{H}$ & - & - & - & - & - \\
\hline & $\begin{array}{l}\text { Automobile } \\
\text { (incl. Taxi) }\end{array}$ & $\mathrm{H}$ & L-H & $\mathrm{H}$ & $\mathrm{H}$ & - & - & - & - & $\mathrm{L}$ \\
\hline & Train & $\mathrm{L}$ & - & - & - & $\mathrm{H}$ & - & - & - & - \\
\hline
\end{tabular}

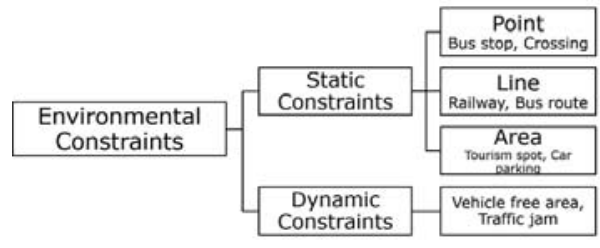

Figure 2: Static and Dynamic Environmental Constraints

are shown in Table 1. For some combinations of velocity and constraints, some of the transportation modes $m$ cannot be available, and these case are indicated by "-" in this table.

When the ranges of velocity of multiple transportation modes are similar, we cannot infer the transportation mode $m_{i}$ of a observation point $i$ by the combination of velocity and environmental constraints $e_{i}$. Therefore, we infer transportation mode $m_{i}$ by using the transportation mode of neighboring observation points and path constraints. In this research, these two are defined as context.

Here, environmental constraints $e$ are regarded as constant with respect to time. However, $e$ could be categorized as dynamic environmental constraints, which are variable with respect to time, and static environmental constraints, which are constant with respect to time (Figure 2). For instance, a road that changes to a vehicle-free street only on weekends is allocated a "road" attribute on weekdays and a "pedestrian road" attribute on the weekends.

In many cases, the location data of the static environmental constraints such as railways or bus routes are provided by governments or private business and can be easily used for research or business. In contrast, it is usually difficult to collect most dynamic environmental constraints like traffic congestion because these data are not measured or disclosed.

This research aims to be accurate enough to infer the transportation mode by using popularly available environmental constraints in as many cities as possible. Therefore, dynamic environmental constraints such as real-time bus locations or traffic congestion data are not used in this research.

\subsection{Overview of the Proposed Method}

An overview of the proposed method is shown in Figure 1.

Before inference, the GPS trajectory data is preprocessed. Because there is measurement error in the GPS trajectory data, this error is removed using the extended Kalman filter and fixed-interval smoothing. GPS trajectory data includes outliers that cannot be removed by the extended Kalman filter and fixed-interval smoothing when the tourist moves underground or indoors. Therefore, outliers are filtered and remove if the tourist velocity is over a threshold $u$.

Tourist velocity is calculated from the coordinates of the GPS trajectory data [9], and velocity class $s_{i}$ is allocated to observation point $i$. Velocity class $s_{i}$ is decided by dividing the velocity range into an adequate number of classes.

Next, the transportation mode of an observation point is temporally labeled using the environmental constraints $e$ First, an $A_{i}$ that indicates the existence of all environmental constraints at each observation point of GPS trajectory data is allocated. The transportation mode of the observation point is temporally labeled based on the combination of velocity class $s_{i}$ and environmental constraints $e_{i}$. Second, the consecutive observation points with the same label are defined as a segment, and the transportation mode of the segment is revised using the context of the neighborhood segments. After revising all the segments, the GPS trajectory is processed based on the new labels, and the procedure is repeated until convergence. Finally, the system outputs the inference as a vector of segments $g_{j}$ that consist of beginning time $b_{j}$, ending time $e_{j}$, and transportation mode $m_{j}$.

\subsection{Preprocessing}

This section details how the system removes the measurement error of a GPS trajectory, calculates the velocity based on this revised trajectory, and allocates a velocity class to each observation point. There is measurement error in the GPS trajectory data because of the propagation velocity of electromagnetic waves as well as influences of the ionization layer and atmosphere, and this error affects the determination of the environmental constraints. Therefore, in this study, the GPS trajectory is approximated using a linear discrete model, and the error is removed by an extended Kalman filter and fixed-interval smoothing [3].

Tourists do not move at a constant velocity, and they sometimes stop moving or change direction quickly. Therefore, the movement of tourists can be considered a non-linear system. However, their movement over a very short interval such as $1 \mathrm{~s}$ can be assumed to be linear. This movement can be approximated as a linear discretetime stochastic system [13]. The tourist status equation, expressed as a non-linear system, and its observation equation are, respectively,

$$
\begin{aligned}
\boldsymbol{x}_{k+1} & =\boldsymbol{F}_{k} \boldsymbol{x}_{k}+\boldsymbol{G}_{k} \boldsymbol{w}_{k} \\
\boldsymbol{y}_{k} & =\boldsymbol{H}_{k} \boldsymbol{x}_{k}+\boldsymbol{v}_{k}, k \geq 0
\end{aligned}
$$

where $\boldsymbol{x}_{k}$ is the true location of the tourist at discrete time $k$, $\boldsymbol{F}_{k}$ and $\boldsymbol{G}_{k}$ are the status transition and control input matrices, respectively, and $\boldsymbol{w}_{k}$ is the process noise. Furthermore, $\boldsymbol{v}_{k}$ in Eq. 3 is observation noise, $\boldsymbol{H}_{k}$ is the observation matrix, and $\boldsymbol{y}_{k}$ is the tourist's location as measured by the GPS at discrete time $k$. Initial status $\boldsymbol{x}_{0}$, system noise $\boldsymbol{w}_{k}$, and observation noise $\boldsymbol{v}_{k}$ are assumed 
to satisfy Eq. 4 and are Gaussian. Note that $\boldsymbol{F}_{k}, \boldsymbol{G}_{k}, \boldsymbol{H}_{k}, \boldsymbol{x}_{0}, \boldsymbol{\Sigma}_{x_{0}}$, $\boldsymbol{\Sigma}_{\boldsymbol{w}_{0}}$, and $\boldsymbol{\Sigma}_{\boldsymbol{x}_{0}}$ are given.

$$
\begin{array}{r}
E\left\{\boldsymbol{w}_{k}\right\}=\mathbf{0}, E\left\{\boldsymbol{v}_{k}\right\}=\mathbf{0}, E\left\{\boldsymbol{x}_{0}\right\}=\overline{\boldsymbol{x}}_{0} \\
E\left\{\left[\boldsymbol{x}_{0}-\overline{\boldsymbol{x}}_{0}\right]\left[\boldsymbol{x}_{0}-\overline{\boldsymbol{x}}_{0}\right]^{\mathrm{T}}\right\}=\sum_{\boldsymbol{x}_{\mathbf{0}}} \\
E\left\{\left[\begin{array}{c}
\boldsymbol{w}_{k} \\
\boldsymbol{v}_{k}
\end{array}\right]\left[\boldsymbol{w}_{k}^{\mathrm{T}}, \boldsymbol{v}_{k}^{\mathrm{T}}\right]\right\}=\left[\begin{array}{rr}
\boldsymbol{\Sigma}_{\boldsymbol{w}_{k}} & \mathbf{0} \\
\mathbf{0} & \sum_{\boldsymbol{v}_{k}}
\end{array}\right] \delta_{k} \\
\sum_{\boldsymbol{v}_{k}}>\mathbf{0}, E\left\{\boldsymbol{x}_{0} \boldsymbol{v}_{k}^{\mathrm{T}}\right\}=\mathbf{0}, E\left\{\boldsymbol{x}_{0} \boldsymbol{w}_{k}^{\mathrm{T}}\right\}=\mathbf{0}
\end{array}
$$

By applying the Kalman filter algorithm to the discrete linear model, we obtain an expanded Kalman filter algorithm. The Kalman filter is a linear stochastic system, where the input is observation value $\boldsymbol{y}_{k}$ and the output is the inference value $\hat{\boldsymbol{x}}_{k \mid k}$ of true location $\boldsymbol{x}_{k}$. The algorithm is given by the following equations.

$$
\begin{aligned}
\hat{\boldsymbol{x}}_{k \mid k} & =\hat{\boldsymbol{x}}_{k \mid k-1}+\boldsymbol{K}_{k}\left(\boldsymbol{y}_{k}-\boldsymbol{H}_{k}\left(\hat{\boldsymbol{x}}_{k \mid k-1}\right)\right) \\
\hat{\boldsymbol{x}}_{k+1 \mid k} & =\boldsymbol{F}\left(\hat{\boldsymbol{x}}_{k \mid k}\right) \\
\boldsymbol{K}_{k} & =\hat{\boldsymbol{\Sigma}}_{k \mid k-1} \boldsymbol{H}^{T}\left(\boldsymbol{H}_{k} \hat{\boldsymbol{\Sigma}}_{k \mid k-1} \boldsymbol{H}_{k}^{T}+\boldsymbol{\Sigma}_{\boldsymbol{v}_{k}}\right)^{-1} \\
\hat{\boldsymbol{\Sigma}}_{k \mid k} & =\hat{\boldsymbol{\Sigma}}_{k \mid k-1}-\boldsymbol{K}_{k} \boldsymbol{H} \hat{\boldsymbol{\Sigma}}_{k \mid k-1} \\
\hat{\boldsymbol{\Sigma}}_{k+1 \mid k} & =\boldsymbol{F}_{k} \hat{\boldsymbol{\Sigma}}_{k \mid k} \boldsymbol{F}_{k}^{T}+\boldsymbol{G}_{k} \boldsymbol{\Sigma}_{\boldsymbol{w}} \boldsymbol{G}_{k}^{T}
\end{aligned}
$$

where $K$ is the Kalman filter gain, and $\hat{\boldsymbol{\Sigma}}$ is the covariance matrix of the estimate error. When a new observation value is gained, the system sequentially calculates a new estimate value. By applying fixed-interval smoothing to $\hat{\boldsymbol{x}}_{k \mid k}$, coordinates are obtained for the velocity calculation. Velocity at observation point $i$ is calculated from distance $D$ between two consecutive observation points

\begin{tabular}{|c|c|c|c|c|}
\hline & Velocity $(\mathrm{m} / \mathrm{s})$ & Car & Walk & Train \\
\hline \multirow[t]{2}{*}{ Low } & $0-2$ & 0.18 & 0.81 & 0.01 \\
\hline & $2-3$ & 0.96 & 0 & 0.04 \\
\hline \multirow{3}{*}{ Middle } & $3-4$ & 0.96 & 0 & 0.04 \\
\hline & $4-6$ & 0.95 & 0 & 0.05 \\
\hline & $6-8$ & 0.96 & 0 & 0.04 \\
\hline \multirow{4}{*}{ High } & $8-10$ & 0.96 & 0 & 0.04 \\
\hline & $10-20$ & 0.94 & 0 & 0.06 \\
\hline & $20-30$ & 0.99 & 0 & 0.01 \\
\hline & $30-50$ & 0 & 0 & 1 \\
\hline
\end{tabular}
$p\left(x_{p}, y_{p}, t_{p}\right), q\left(x_{q}, y_{q}, t_{q}\right)$ and transit time $t_{q}-t_{p}$. Distance $D$ is calculated using Hubeny's distance formula, calculated as follows.

$$
\begin{aligned}
D(p, q) & =\sqrt{\left(d_{y} Q\right)^{2}+\left(N d_{x} \cos \left(a_{y}\right)\right)^{2}} \\
d_{y} & =y_{p}-y_{q} \\
d_{x} & =x_{p}-x_{q} \\
a_{y} & =\frac{y_{p}+y_{q}}{2}
\end{aligned}
$$

In Eq. 6, $Q$ is the Earth's radius of curvature along the (northsouth) meridian, and $N$ is the radius of the curvature in the prime vertical. Velocity class $s_{i}$ of observation point $i$ is allocated based on the obtained velocity.

\subsection{Inference Using Observation Points}

In this section, observation point $i$ is temporally labeled with transportation mode $m_{i}$ based on the combination of velocity class $s_{i}$ and environmental constraints $e_{i}$.

We allocate $A_{i}$, which indicates the existence of all environmental constraints $e$, to an observation point $i$ as $i\left(x_{i}, y_{i}, t_{i}, s_{i}, A_{i}\right)$, and calculate the probability $P_{i}(m)$ of each transportation mode corresponding to the environmental constraint $e_{i}$ at observation point $i$. The transportation mode $m$ that maximizes $P_{i}(m)$ temporally labels $i$.

Probability $P_{i}$ is calculated using manually labeled training data. Because $P_{i}$ varies depending on the environmental constraints $e_{i}$, even in the same velocity class, $P_{i}$ is calculated as follows.

$$
P_{i}(m ; s, e)=\frac{r_{m, e}(s)}{\sum_{k \in M} r_{k, e}(s)}, m \in M, e \in E
$$

In Eq. $7, s$ is the velocity class of the observation point, $m$ is a transportation mode, $M$ is the set of all transportation modes, $e$ is an environmental constraint, $E$ is the set of all environmental constraints, $r_{m, e}(s)$ is sum of observation points that are allocated velocity class $s$ and are located in environmental constraints $e$ in the training data. This probability for various transportation modes is listed in Table 2.

\subsection{Revision by Segment with Context}

Next, temporal labels are revised by applying the context. To apply the context, a segment is created by merging consecutive observation points that have the same label. The context is then applied to the segment. Different inference techniques are applied depending on the environmental constraints, because the temporal and topographical ranges of velocity change depending on the applied velocity constraints.

The point-based environmental constraints $e_{k}^{p}$ constrain the velocity of observation points that are located in geographical neighborhood. The number of constrained observation points is relatively small. There are two cases of velocity and transportation mode change patterns around point-based environmental constraint $e_{k}^{p}$. In the first case, only the velocity changes. Transportation mode $m_{i}$ of segment $g_{i}$ that includes the constrained observation points is the same as transportation modes $m_{i \pm 1}=m_{i}$ of neighborhood segments $g_{i \pm 1}$. In the second case, both velocity and transportation mode change. Transportation mode $m_{i}$ of segment $g_{i}$ is not the same as transportation modes $m_{i \pm 1}$ of neighborhood segments $g_{i \pm 1}$. The velocity change should be determined around point-based environmental constraint $e_{k}^{p}$ according to the transportation mode changes or not for the revised label. The difference in these cases is that the transportation modes change or not depending on the number $d_{i}$ of observation points of segment $g_{i}$. Therefore, $d_{i}$ is scrutinized. The distribution of the number of observation points $d$ can be determined from the training data. If $d_{i}$ is subject to the distribution, the velocity change in segment $g_{i}$ is a temporal velocity change caused by the point-based environmental constraints. The transportation mode can then be inferred as $m_{i \pm 1}$. If $d_{i}$ is not subject to the distribution, the velocity change in segment $g_{i}$ is caused by a transportation mode change.

The line-based environmental constraints constrains the velocity of additional observation points. The number of constrained observation points is more than those caused by point-based environmental constraints. In a segment $g_{i}$ that has its velocity constrained by line-based environmental constraints $e_{k}^{s}$, if the transportation modes of the neighboring segments $g_{i \pm 1}$ are the same $m_{k}$ and $m_{k}$ does not 


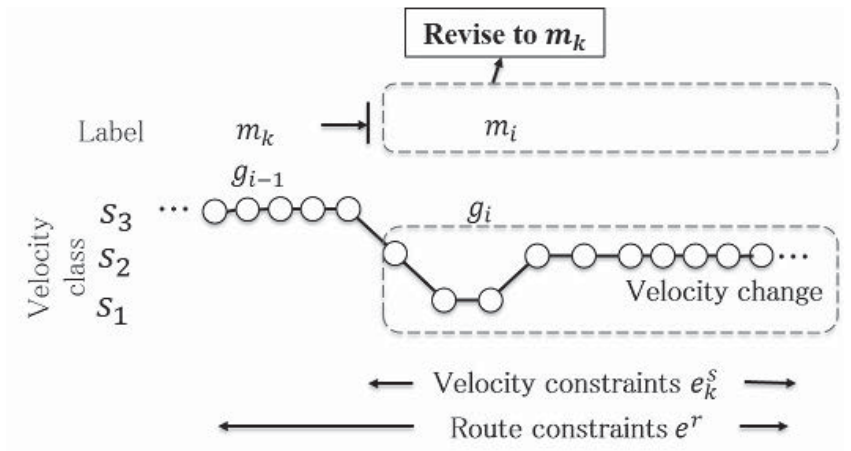

Figure 3: Transportation Mode Revision from Linear Environmental Constraints

conflict with $e^{r}$ in the consecutive segments $g_{i-1}, g_{i}$, and $g_{i+1}$, the transportation mode of segment $g_{i}$, is inferred to be $m_{k}$. This is illustrated in Figure 3. Stations and railways as well as bus stops and public buses are examples of combinations of velocity constraints $e_{k}^{s}$ that constrain certain transportation modes $m_{k}$.

Most of the area-based environmental constraints limit the usage of transportation modes. Therefore, by applying the area-based environmental constraint, the available transportation modes can be limited.

\section{EXPERIMENTS}

\subsection{Overview of Experiments}

To measure the performance of the proposed algorithm, the transportation mode with GPS trajectory data of actual tourists was inferred. For comparison, the transportation mode was inferred using only velocity without environmental constraints. The same dataset with the error removed was used for the comparison test.

Participants were student groups and their escorts during group activities of their school trips. The group leaders of student groups carried smartphones that had GPS loggers installed. The number of participants in the study was 60 . Hence, the dataset was made up of the data of 60 GPS trajectories collected from the participants. The dataset included 1,069,101 observation points after outlier removal. The participants moved in over the course of a day with GPS supported smartphones, and the trajectory data was collected by the GPS logger application. As the GPS logger application, the ETSS application was used[5]. Participants moved over a period of 6 to 10 hours in a day, from 8:00 AM until 7:00 PM. As the survey area, Kyoto, Osaka, and Nara, where many students go on school trips, were selected. These cities are located in the Kinki area in Japan. The transportation modes $m$ that the participants could use were walking, taxis, public buses, and trains. No one used bicycles, motorbikes, rental cars, or private cars. The result was evaluated by a leave-one-out cross validation method. The correct classification for each observation point was determined by human verification.

\subsection{Environmental Constraints}

For the environmental constraints, the spatial information of bus routes, railways, and pedestrian areas were used. For stations, railways, and motorways, transportation network data was acquired from a private map company. For bus routes, public bus route data from digital national land information provided by the Geospatial Information Authority of Japan was used. For pedestrian areas, the spatial data of tourist spots from Kyoto City local government were obtained. All data was provided in shapefile format, which is generally used for geoinformation system science.

A buffer was applied to point and line-based environmental constraints for convenience. A 40-meter buffer was used for railways and motorways, and a 20-meter buffer was used for bus routes. No buffer was used for pedestrian areas.

According to the location in physical space, multiple environmental constraints can exist in the same location. In this experiment, priority is given to environmental constraints, and the probability $P_{i}\left(m_{h}\right)$ of the most prioritized environmental constraint $m_{h}$ is allocated to observation point $i$. The order of the priority is shown in Table 3.

1. Railways
2. Bus routes
3. Motorways
4. Pedestrian areas

Table 3: Order of Priority

\subsection{Thresholds for Preprocessing}

The velocity threshold $u$ for outlier removal of GPS trajectory data was set to 50 meter/s. This was determined based on the velocity of the fastest transportation in the survey area.

\subsection{Car Mode}

Public buses and taxis operate at a similar velocity and hence, it is difficult to discriminate between them by using the velocity of observation points. Therefore, in this experiment, after inferring the mode to be car mode, which consists of both taxi and public bus modes, both modes were split by applying path and velocity constraints to the segments. Public bus routes were used as the route constraint, and bus stop were used as the velocity constraint.

Although the Geospatial Information Authority of Japan provides bus stop locations, the accuracy is not sufficient for use as a velocity constraint. Hence, bus stop locations are inferred from the training data. These locations were inferred to be where the public buses stopped in the training data. A stopping event is determined when the public bus moves at under $2 \mathrm{~m} / \mathrm{s}$.

\section{RESULTS}

The results of the experiment are shown in Tables 4 and 5. Table 4 shows the result when public bus mode and taxi mode were inferred separately. Table 5 shows the result when public bus mode and taxi mode were inferred as one mode. The results show that the total recall for the four-mode case was $90.8 \%$ and the total recall of the three-mode case was $94.4 \%$. The recall of public bus, taxi, train, and walking modes were $57.7 \%, 65.8 \%, 91.6 \%$, and $98.2 \%$, respectively.

The total recalls of the comparison method, were $80.4 \%$ (four modes) and $85.0 \%$ (three modes), as shown in Table 6 and Table 7. Although the the comparison method inferred 7,231 segments (four modes) and 6,979 segments (three modes), the number of segments inferred by the proposed method for four and three modes was 884 and 850 , respectively. The true number of segments is 515 .

For helping understanding the results of the experiments, we illustrate the inference result of participant \#26 in Figure 4. Yellow lines mean the taxi mode, light blue lines mean the bus mode, green lines mean The upper figure shows the result of inference, and lower figure shows correct modes. The recall rate of the participant \#26 trajectory is $94.8 \%$, and the inferred number of segments 
Table 4: Four-mode Recall of the Proposed Method (Recall 90.8\%)

\begin{tabular}{|l|l|cccc|}
\hline \multicolumn{2}{|l|}{} & \multicolumn{4}{|c|}{ True } \\
\hline & & Bus & Taxi & Train & Walk \\
& & $\mathrm{n}=60,808$ & $\mathrm{n}=152,798$ & $\mathrm{n}=86,627$ & $\mathrm{n}=768,868$ \\
\hline \multirow{3}{*}{ Infer } & Bus & $57.7 \%$ & $12.3 \%$ & $0.0 \%$ & $0.4 \%$ \\
-red & Taxi & $32.3 \%$ & $65.8 \%$ & $1.1 \%$ & $1.1 \%$ \\
& Train & $0.3 \%$ & $3.7 \%$ & $91.6 \%$ & $0.3 \%$ \\
& Walk & $9.7 \%$ & $18.2 \%$ & $7.2 \%$ & $98.2 \%$ \\
\hline
\end{tabular}

Table 5: Three-mode Recall of the Proposed Method (Recall 94.4\%)

\begin{tabular}{|c|c|ccc|}
\hline \multicolumn{2}{|c|}{} & \multicolumn{3}{|c|}{ True } \\
\hline \multirow{3}{*}{ Inferred } & Car & Train & Walk \\
& & $\mathrm{n}=213,606$ & $\mathrm{n}=86,627$ & $\mathrm{n}=768,868$ \\
\hline \multirow{3}{*}{} & Car & $81.5 \%$ & $1.1 \%$ & $1.5 \%$ \\
& Train & $2.7 \%$ & $91.6 \%$ & $0.3 \%$ \\
& Walk & $15.8 \%$ & $7.2 \%$ & $98.2 \%$ \\
\hline
\end{tabular}

Table 6: Four-mode Recall of the Velocity-only Method (Recall 80.4\%)

\begin{tabular}{|l|l|cccc|}
\hline \multicolumn{2}{|c|}{} & \multicolumn{4}{|c|}{ True } \\
\hline & & $\begin{array}{c}\text { Bus } \\
\mathrm{n}=60,808\end{array}$ & $\begin{array}{c}\text { Taxi } \\
\mathrm{n}=152,798\end{array}$ & $\begin{array}{c}\text { Train } \\
\mathrm{n}=86,627\end{array}$ & $\begin{array}{c}\text { Walk } \\
\mathrm{n}=768,868\end{array}$ \\
\hline \multirow{3}{*}{ Infer } & Bus & $0.0 \%$ & $0.0 \%$ & $0.0 \%$ & $0.0 \%$ \\
-red & Taxi & $54.5 \%$ & $50.1 \%$ & $49.7 \%$ & $1.7 \%$ \\
& Train & $0.2 \%$ & $15.7 \%$ & $31.9 \%$ & $0.1 \%$ \\
& Walk & $45.3 \%$ & $34.2 \%$ & $18.4 \%$ & $98.2 \%$ \\
\hline
\end{tabular}

Table 7: Three-mode Recall of the Velocity-only Method (Recall 85.0\%)

\begin{tabular}{|c|l|ccc|}
\hline \multicolumn{2}{|c|}{} & \multicolumn{3}{|c|}{ True } \\
\hline \multirow{3}{*}{ Inferred } & Car & Train & Walk \\
& & $\mathrm{n}=213,606$ & $\mathrm{n}=86,627$ & $\mathrm{n}=768,868$ \\
\hline \multirow{3}{*}{} & Car & $59.2 \%$ & $50.0 \%$ & $1.7 \%$ \\
& Train & $3.4 \%$ & $31.6 \%$ & $0.1 \%$ \\
& Walk & $37.4 \%$ & $18.4 \%$ & $98.2 \%$ \\
\hline
\end{tabular}

is 30 . The currect number of segments is 15 . The participant \#26 used all 4 transportation modes during the trip between 8:00 AM and 6:00 PM. The participant \#26 started from a hotel in Otsu that is located in right area of the figure, and after walked for a while, went to Otsu station by taxi. From Otsu station, the participant \#26 rode on a train to Kyoto station that is located in left central area of the figure. During the movement, the trajectory was not measured two times because of two tunnels among Otsu station and Kyoto station. In Kyoto city area, the participant \#26 went to a downtown area by train and went around by his foot for a while. After stroll around the downtown area, the participant \#26 went to a animation fun shop that is located in the south area of Kyoto city by train, and returned to the hotel by train and taxi.

Figure 5 shows all points where the proposed method could not inferred correctly. Points located near Otsu station in the right side

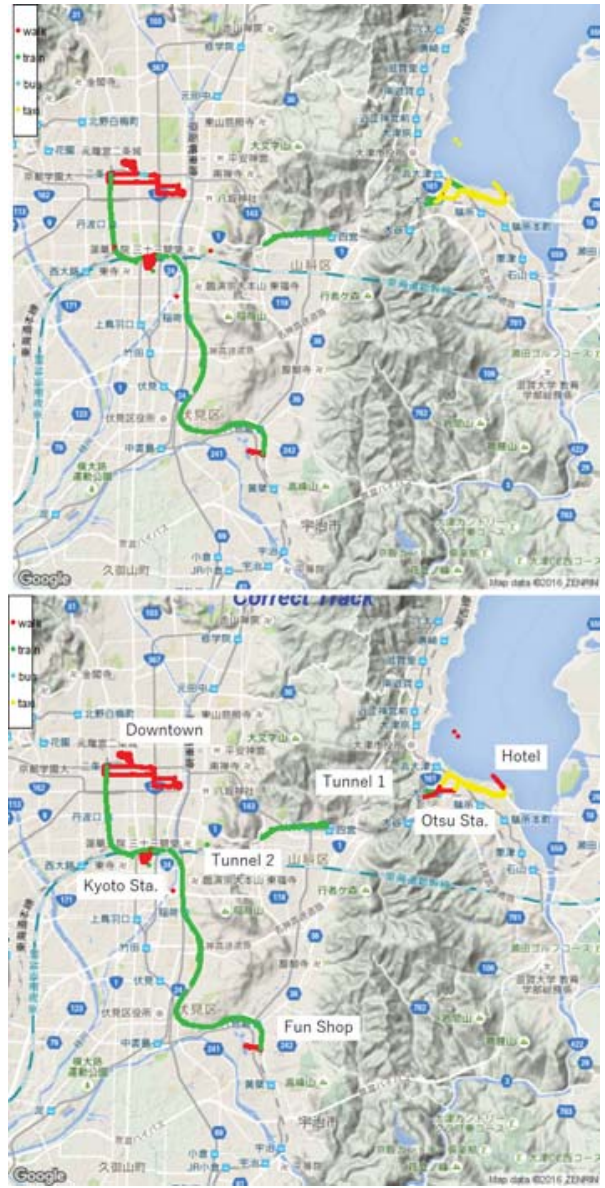

Figure 4: Example of Results (Participant \#26)

(The upper figure shows the inference results. The lower figure shows the correct modes. )

of the Figure 5 were inferred as taxi while the correct modes are walk. We guess that these failures were caused by low accuracy of GPS data. Because there are many buildings around Otsu station, accuracy of GPS data tends to be lower. Some failures along with railways to west side of Kyoto station would be caused by some pedestrian areas near the trajectory. In the downtown area, some points were inferred as bus while the correct transportation modes are walk. The participant \#26 walked along with a public bus route for around 4 minutes at a quick pace near here, therefore, the system inferred as bus.

\section{DISCUSSION}

As the results confirm, a $90.8 \%$ recall was achieved by applying the proposed method to GPS trajectory data for four modes.As described in Subsection 3.3, the proposed method consists of two parts excluding the preprocessing process. Here, I explain the process of the inference step by step.

First, the recall of three-mode temporal labeling to each observation point based on the velocity class and environmental constraints is $88.9 \%$. The number of segments is 6,798 . The temporal labeling with environmental constraints resulted in an improvement of 3.9\% over the velocity-only method (three modes inference, $85.0 \%$ ). The numbers of inferred segments are almost the same. Next, by revis- 


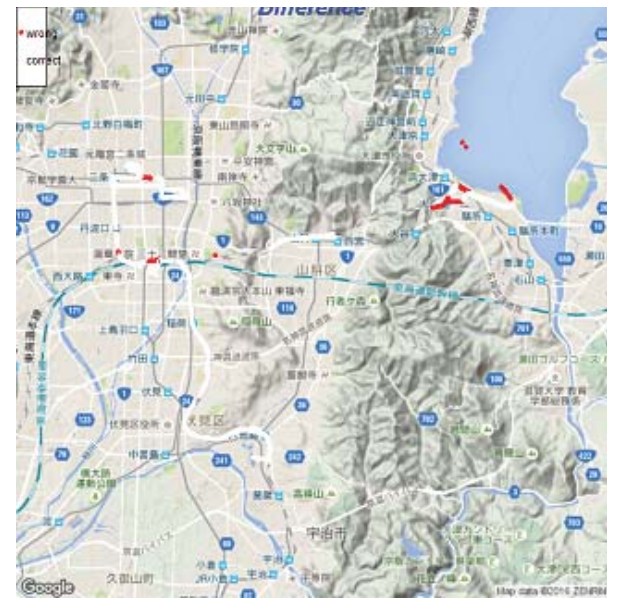

Figure 5: Inference Failure Points (Participant \#26) (Red points mean observation points that were not inferred correctly.)

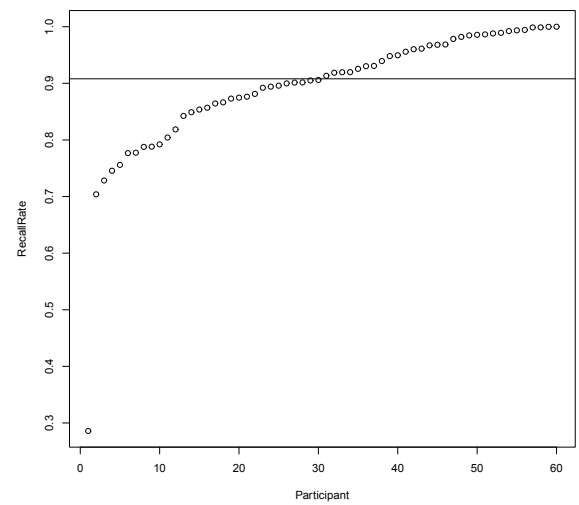

Figure 6: Recalls of All Participants' Trajectories (A horizontal line means the total recall of all trajectories $(90.8 \%))$

ing the label of segments using context, $90.8 \%$ recall is acquired. The revision by context resulted in an improvement of $10.4 \%$ over the comparison method (four modes inference, 80.4\%), and decreases the number of segment by 6,347. The proposed method lowers the number of segments because small segments are correctly merged into neighboring segments by considering the change of velocity caused by the point-based environmental constraints.

For improvement of the proposed inference method, we discuss failures of the inference here. Figure 6 shows recalls of all participants' trajectories. The worst inference is for participant \#43. The reason why the trajectory was not inferred correctly is that the trajectory was not measured consecutively as shown in Figure 7.

Typical failure is occurred among buses and taxies like the participant \#54 trajectory shown in Figure 8. In this case, although the participant \#54 used taxi, some taxi segments are inferred as bus. Because the segments are on public bus paths, we can not determine the transportation mode correctly with features that we used for this experiments. It is expected that it will soon become easy to

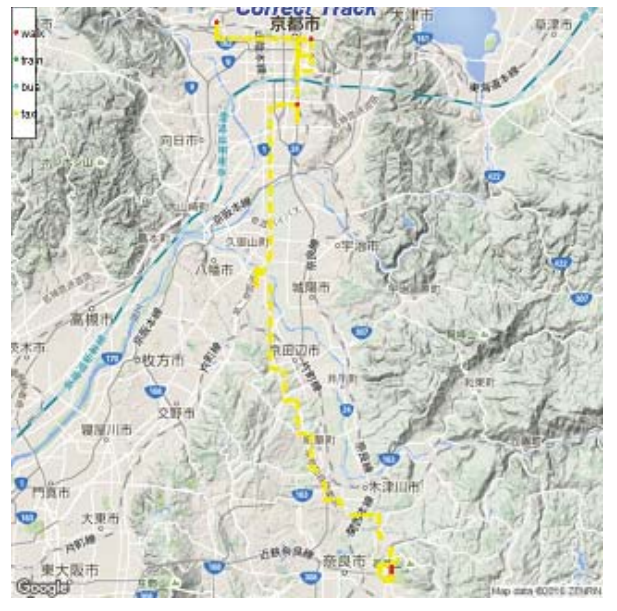

Figure 7: A Trajectory that was not Measured Consecutively (participant \#43)

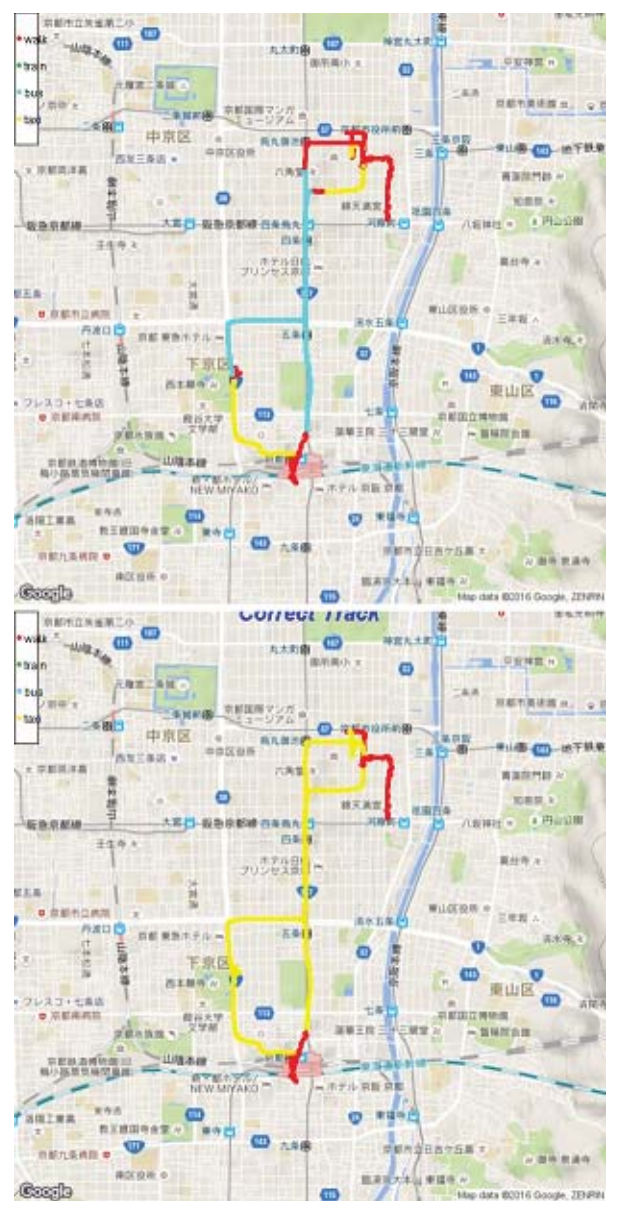

Figure 8: Failure among Bus and Taxi (participant \#54) (The upper figure shows the inference results. The lower figure shows the correct modes. )

use public geospatial data such as bus, bus stop, and tourism spot location data that are now difficult to acquire. 
In this research, the proposed method was evaluated by actual GPS trajectory data that included four transportation modes. Other GPS trajectory data including other transportation modes was not evaluated. Bicycle mode may be easily inferred because of its distinct velocity range. Other modes such as motorbike, rental car, and private car modes may be difficult to determine because of the similarity of the average velocity of these transportation modes. In order to infer these transportation modes, car parking location data, where only these modes are allowed to stop, could be useful. In addition, most tourists who use private cars may not use other transportation modes such as taxis and public buses. Similarly, there are relationships among some transportation modes that are simultaneously used. Such context of transportation mode usage in daily movement could improve the inference.

GPS accuracy would also improve the inference of the proposed method. In cases where a significant error may occur because of multilevel streets or other reasons, adequate environmental factors cannot be attached to the observation points. Map matching technology that can specify the tourist paths using traffic network data would be useful for car trajectories. However, people who move on foot sometimes walk along under $4 \mathrm{~m}$ wide roads, and it is difficult to apply map matching technology to such narrow roads.

In addition, dynamic environmental constraints like traffic congestion are considered to be one of the factors that decrease the inference accuracy. Traffic congestion affects the velocity of cars, and low velocity observation points may be inferred as walking mode without traffic congestion information. This study does not use the traffic congestion information because it is difficult to acquire.

\section{CONCLUSION}

This paper proposes a transportation mode inference method that uses environmental constraints. The results of the experiments indicate satisfactory levels of accuracy for the practical application of tourist activity support. As shown in Figure 4, the results of the proposed method match human judgment, and it has enough accuracy for practical usage. By determining the transportation mode, social resources will be allocated efficiently both in disaster and non-disaster situations. In addition, the result can be used for touristic statistics, which is helpful for tourist destination management organizations. However, considering the application for tourist activity support or tourism spot recommendation, the development of an online transportation mode inference method should be studied in future. In case of the online transportation mode inference, the end of the segment is not determined from past data because the future data is not available. Therefore, the relationship between the transportation mode of the previous segment and the elapsed time of the current segment could also be used for analysis.

\section{REFERENCES}

[1] M. AOKI, S. SEKO, M. NISHINO, T. YAMADA, S. MUTO, and M. ABE. An estimating method for activity modes using location data. IPSJ SIG Notes, 67:7-12, jul 2008.

[2] A. Bolbol, T. Cheng, I. Tsapakis, and J. Haworth. Inferring hybrid transportation modes from sparse gps data using a moving window svm classification. Computers, Environment and Urban Systems, 36(6):526-537, 2012.

[3] J. Durbin and S. J. Koopman. Time series analysis by state space methods. Number 38. Oxford University Press, 2012.

[4] S. Hemminki, P. Nurmi, and S. Tarkoma. Accelerometer-based transportation mode detection on smartphones. In Proceedings of the 11th ACM Conference on Embedded Networked Sensor Systems, page 13. ACM, 2013.
[5] H. Kasahara, K. Kurumatani, M. Mori, M. Mukunoki, and M. Minoh. Evacuation support and safety confirmation sharing in disaster situations for school trips by mobile information system. Information Technology $\mathcal{E}$ Tourism, 14(3):197-217, 2014.

[6] A. Kinoshita, A. Takasu, K. Aihara, J. Ishii, H. Kurasawa, H. Sato, M. Nakamura, and J. Adachi. Gps trajectory data enrichment based on a latent statistical model. In Proceedings of the 5th International Conference on Pattern Recognition Applications and Methods, pages 255-262, 2016.

[7] L. Liao, D. Fox, and H. Kautz. Extracting places and activities from gps traces using hierarchical conditional random fields. The International Journal of Robotics Research, 26(1):119-134, 2007.

[8] D. Montoya, S. Abiteboul, and P. Senellart. Hup-me: Inferring and reconciling a timeline of user activity from rich smartphone data. In Proceedings of the 23rd SIGSPATIAL International Conference on Advances in Geographic Information Systems, GIS '15, pages 62:1-62:4, New York, NY, USA, 2015. ACM.

[9] M. Nagao, H. Kawamura, M. Yamamoto, and A. Ohuchi. Analysis of circular tour activity based on gps log. Information and Communication Technologies in Tourism 2006, pages 87-98, 2006.

[10] H. Ohashi, T. Akiyama, M. Yamamoto, and A. Sato. Modality classification method based on the model of vibration generation while vehicles are running. In Proceedings of the Sixth ACM SIGSPATIAL International Workshop on Computational Transportation Science, page 37. ACM, 2013.

[11] R. C. Shah, C.-y. Wan, H. Lu, and L. Nachman. Classifying the mode of transportation on mobile phones using gis information. In Proceedings of the 2014 ACM International Joint Conference on Pervasive and Ubiquitous Computing, pages 225-229. ACM, 2014.

[12] L. Stenneth, O. Wolfson, P. S. Yu, and B. Xu. Transportation mode detection using mobile phones and gis information. In Proceedings of the 19th ACM SIGSPATIAL International Conference on Advances in Geographic Information Systems, pages 54-63. ACM, 2011.

[13] N. Tokunari, S. Minoru, I. Satoshi, and O. Naoki. Smoothing gps data using extended kalman filter. The Japan Society Applied Electromagnetics and Mechanics, 19(3):591-598, 2011.

[14] Z. Yan, D. Chakraborty, C. Parent, S. Spaccapietra, and K. Aberer. Semantic trajectories: Mobility data computation and annotation. ACM Transactions on Intelligent Systems and Technology (TIST), 4(3):49, 2013.

[15] M.-C. Yu, T. Yu, S.-C. Wang, C.-J. Lin, and E. Y. Chang. Big data small footprint: the design of a low-power classifier for detecting transportation modes. Proceedings of the VLDB Endowment, 7(13):1429-1440, 2014.

[16] V. W. Zheng, Y. Zheng, X. Xie, and Q. Yang. Collaborative location and activity recommendations with gps history data. Proceedings of the 19th international conference on World Wide Web, pages 1029-1038, 2010.

[17] Y. Zheng, Y. Chen, Q. Li, X. Xie, and W.-Y. Ma. Understanding transportation modes based on gps data for web applications. ACM Transactions on the Web (TWEB), $4(1): 1,2010$ 